\title{
THE PROPOSAL OF THE CLASSIFICATION SYSTEM FOR PARTS PRODUCED BY CNC MACHINES
}

\section{Vladimir Simna, Peter Pokorny \\ Slovak University of Technology, Faculty of Materials Science and Technology in Trnava, Institute of Production Technologies, Department of Machining and Assembly Trnava, Slovak Republic \\ e-mail: peter_pokorny@stuba.sk}

This paper deals with the design of the classification system which task will be component classification from view of manufacturability by CNC machines. Each component can be described by distribution and orientation features and surfaces witch located in the component, but it is also necessary to take into account what relations exist between these elements. For the purposes of computer representations of CNC machine seems to be most efficient solution describe the kinematic characteristics by mathematical formulas which clearly define the option of the kinematics of CNC machine. These data can be processed by the theory of forward or inverse kinematics.

Keywords:

classification, features, inverse kinematic, CAPP, basis function

\section{Introduction}

Computer-aided process planning (CAPP) is considered to be the bridge that joins computer-aided design (CAD) and computer-aided manufacturing (CAM). One of the most important aspects of CAPP is automatic feature extraction, which is a procedure that aids in the generation of process plans automatically [Shu-Chu 1995].

An important aspect of manufacturing automation is the translation of data defining a given part into the data required for the actual manufacturing of the part. Computer-aided process planning (CAPP) serves as the vital link between design and production to convert raw material into finished product [Chang 1991].

A part feature is defined as a specific geometric configuration formed on the surface, edge or comer of a workpiece. It is a geometrical shape that can be generated through a specific machining process [Salomons 1988].

Generally CAD system allows realising required shape surface quality relatively easily [Fabian 2010].

For example, part features that are flat can be generated through milling or shaping processes. During the last few years, research has focused on the extraction of features from CAD files. The literature shows a number of algorithms that have been proposed for extracting machining features from a part design drawing [Cunningham 1988].

Eight of the most important approaches are as follows:

1. section techniques.;

2. convex-hull algorithm;

3. cell decomposition;

4. geometric reasoning;

5. pattern matching;

6. entity growing;

7. volume decomposition;

8. recognition from Constructive Solid Geometry (CSG) trees.

A 5-axis CNC machine tool totally has 5 degrees of freedom (DOF) which consist of 2 rotational axes (R) and 3 translational axes $(T)$. The relative movable system, which consists of two or more bodies such as movement axes linked by kinematic pairs, is called as the kinematic chain. The system from spindle motor to tool is the tool kinematic chain and the one from feed motor to worktable is the workpiece kinematic chain. According to configurations and sequences of rotational axes and translational axes on the tool kinematic chain and workpiece kinematic chain, A 5-axis CNC machine tool has 360 types of kinematic chain. Whereas a 5-axis CNC machine tool with a universal head named RR machine can be classified as 20 kinematic chain types [Bohez 2002].

The problem of components classification in view of their production on CNC machines is specific be taken into consideration that two separate units of information and combine them to find the specific criteria by which the element is classified. The first group of information are information about the modeled components, such as its size, orientation in space, the number and type of elements which are in the part. Any component can be considered as a system where the elements are located in the part between them clearly defined session.

The second group of information supplied to the classification process are information about the production possibilities of CNC machines. Before it is possible to classify component is necessary to obtain information (data), by which the component is defined.

\section{Extracting information} about classified components

The most preferred method of representation components are data which have tree structure because using these data is relatively easy to determine the relation between entities which are in the part and then to determine the type of element to the entities represent. A possible structure of the CAD data shows (Fig. 1). This figure shows the structure of the data in the format of STEP AP 214

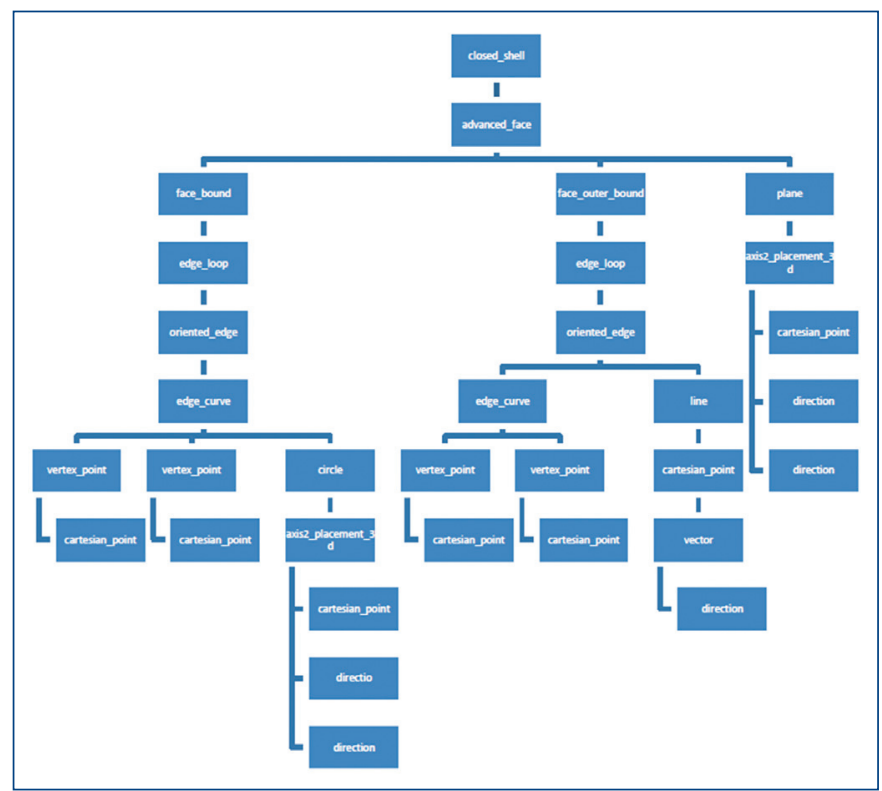

Figure 1. A possible structure of the $C A D$ data

\subsection{Orientation and location of the elements}

In the case when the information about which elements are in the part are known is necessary to identify their orientation and location. For the identification of these parameters is effective to establish a second (local) coordinate system which will be assigned to the element and then find the relations between the global coordinate system respectively between components coordinate system and the local coordinate system thus coordinate system of the element. This situation is shown in (Fig. 2). 


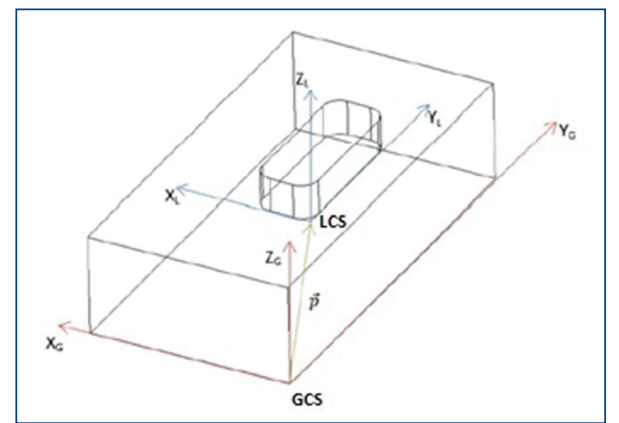

Figure 2. Choice of the coordinate systems of component and feature

In (Fig. 2) are shown coordinate systems and vector $p$ which reflects the shift of global and local coordinate system. Case where the LSS to the GSS only shifted, but also rotated to GSS is shown in (Fig. 3).

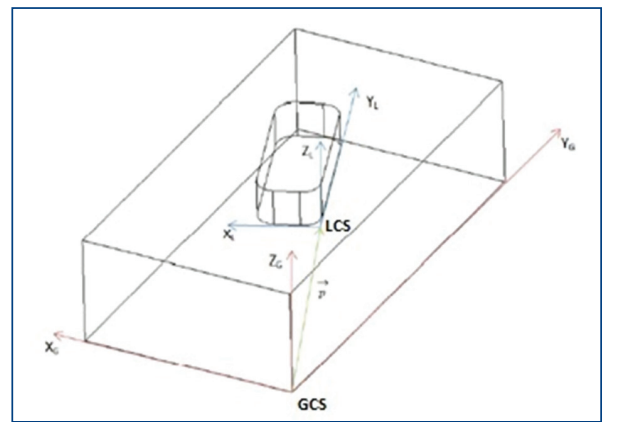

Figure 3. Illustration of the coordinate systems when the feature is rotated

Positioning element shown in (Fig. 3) is not already so clear as in (Fig. 2) and will necessary to establish transformation equations that determine the relationship of GSS to LSS. In this particular case, the element rotated angle $\gamma$ round $Z$ axis (1).

$$
\left[\begin{array}{l}
X_{g} \\
Y_{g} \\
Z_{g}
\end{array}\right]=R_{Z, \gamma} \cdot\left[\begin{array}{l}
X_{L} \\
Y_{L} \\
Z_{L}
\end{array}\right]+\left[\begin{array}{c}
p_{X} \\
p_{Y} \\
p_{Z}
\end{array}\right]
$$

Where $R_{z, Y}$ is the rotation matrix that have in this case form (2).

$$
R_{Z, \gamma}=\left[\begin{array}{ccc}
\cos \gamma & -\sin \gamma & 0 \\
\sin \gamma & \cos \gamma & 0 \\
0 & 0 & 1
\end{array}\right]
$$

\section{Determining the properties of bspline surface}

In STEP 214 the B-spline surface can be defined as it is shown (Fig. 4) In a similar manner is defined Bezier surface or NURBS surface. The first line defines the type of surface numerical values in brackets in the first line define degree of curve which surface consists of (in this case 3,3$)$. The second to fifth line defines network of the control points that describe surface. Seventh and eighth row defines the number of nodal values vector for the curve. In the ninth and tenth row absolute values are expressed. According to the definition of B-spline surfaces there should be used method of deposit where the relationship between the values will be maintained.

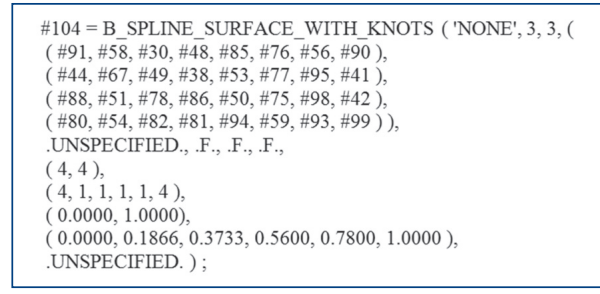

Figure 4. B-spline surface definition in STEP 214
The definition of B-spline surface in STEP 214 does not always have the same structure. It depends on the number of control points and values of nodal vectors. Before we determine the properties of surface we need to know the structure of data. One possible way is to count the number of rows and find what type of information is stored in row. When determining the properties of B-spline surface the most important task is to find values of the basis functions. The basis function is defined by (3) and (4).

$$
\begin{gathered}
N_{i}^{k}(t)=\frac{u-t_{i}}{t_{i+k}-t_{i}} N_{i}^{k-1}(t)+\frac{t_{i+k+1}-u}{t_{i+k+1}-t_{i+1}} N_{i+1}^{k-1} \\
N_{i, 1}(u)=\left\{\begin{array}{c}
1 \text { If } t_{i} \leq u \leq t_{i+1} \\
0 \text { otherwise }
\end{array}\right.
\end{gathered}
$$

To compute the basis functions is used class called b_spline which define the method called basis_unction. The basis functions are computed through cycles which are controlled by data from input STEP file. In this case there can be seen the advantages of object oriented programming because formula to compute derivate of basis function is similar to formula to compute basis function and therefore the principle of heredity can be used.

\subsection{Computation of the surface parameters}

Important information for each surface is the coordinates of normal vector in any point of the surface. B-spline normal vector is defined by (5).

$$
\vec{N}=\frac{d P}{d u} \times \frac{d P}{d v}
$$

Where and are the tangents. The tangents are defined by (6) and (7).

$$
\begin{aligned}
& \frac{d P}{d u}=\sum_{i=1}^{N} \sum_{j=1}^{M} P_{i, j} N_{j, i}(v) \frac{d N_{i, k}(u)}{d u} \\
& \frac{d P}{d v}=\sum_{i=1}^{N} \sum_{j=1}^{M} P_{i, j} N_{i, k}(u) \frac{d N_{i, j}(v)}{d v}
\end{aligned}
$$

Another important information is that about points which are located in the surface. This point is defined by (8).

$$
\mathrm{P}(\mathrm{u})=\sum_{i=0}^{N} \sum_{j=0}^{M} P_{i, j} N_{i, k}(u) N_{j, i}(v)
$$

In the (Fig. 5) we can see the principle for calculating surface properties. Parameters $t_{u, l^{\prime}}, t_{v, l}, P(u)$ and $P(v)$ are obtained directly from the CAD data. $\mathrm{N}_{\text {i, }}$ and $\mathrm{N}_{i, j}$ are the methods for computation surface basis functions. Next method is method for computing derivative of

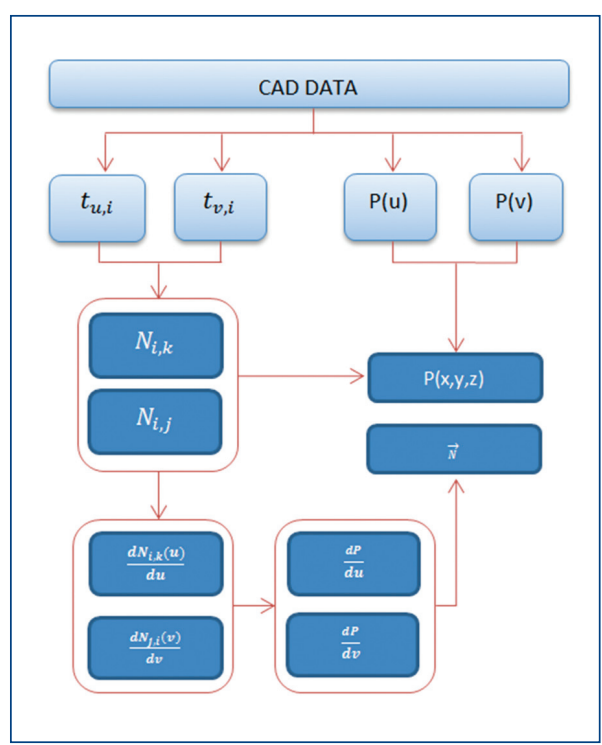

Figure 5. The principal for calculating the properties of b-spline surfaces 
basis functions and the last method is used for tangent computation. Using these three methods, we can calculate the normal vector. These methods must be called in the correct order. To calculate point coordinates we have to call only one method to compute the basis functions. Using this set of methods all properties of the surface are known. All methods are defined in class called b_spline.

\section{Kinematic structures of CNC Machines}

Unlike a feed forward kinematics for the calculation of the inverse kinematics is the task to calculate the parameters of the rotation angles of the axes based on the position of the end spindle point. In this case, the spindle position and orientation of the tool. For a correct expression of the kinematic properties of the machine will need to introduce different coordinate systems and describe the relations between them. An example of such systems can be seen in (Fig. 6).

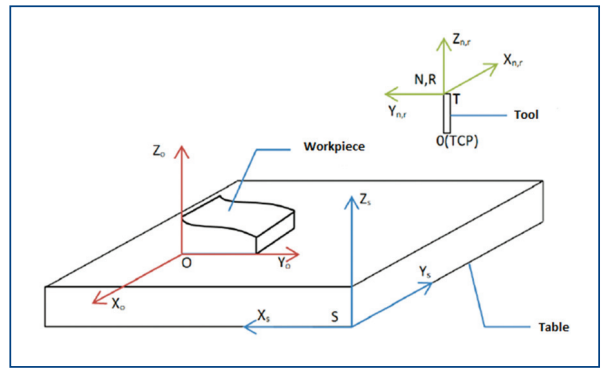

Figure 6. Example of a coordinate system in Initial time

In the (Fig. 6) are see four coordinate systems called, M,W,T, R, respectively coordinate system of the machine, workpiece, tool and reference coordinate system. In the initial time coordinate system $\mathrm{N}$ and $\mathrm{R}$ have the same position. The tool is described by two parameters and it is the point $O$, which represents the tool center point and by the vector $T$, which for clarity is not plotted. These two parameters are described as:

$$
\begin{aligned}
& O=\left[\begin{array}{llll}
0 & 0 & -L & 1
\end{array}\right]^{\top} \\
& T=\left[\begin{array}{llll}
0 & 0 & 1 & 0
\end{array}\right]^{\top}
\end{aligned}
$$

Where $L$ is the length of the center of coordinate system $N$ and the point 0 (TCP). In (Fig. 7) can be seen the position of the coordinate systems in Machining time when the coordinate systems $\mathrm{N}$ and $\mathrm{R}$ have the same position.

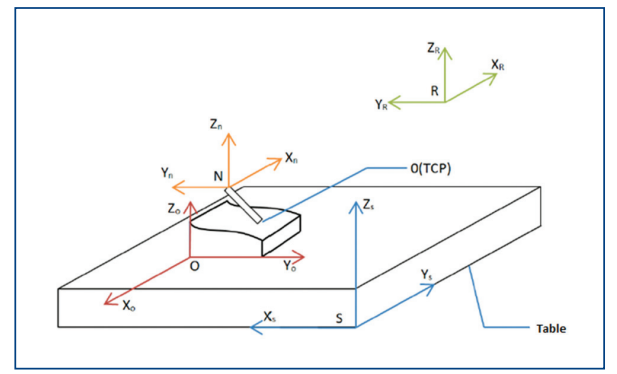

Figure 7. Example of a coordinate system in Machining time

For the kinematic structure "universal head" is characteristic the tool can rotate about axes $A$ and $C$. In this case, it is possible to point $O n$ a vector Tn described as follows:

$O_{n}=\left[\begin{array}{cccc}\cos (C) & -\sin (C) & 0 & 0 \\ \sin (C) & \cos (C) & 0 & 0 \\ 0 & 0 & 1 & 0 \\ 0 & 0 & 0 & 1\end{array}\right]\left[\begin{array}{cccc}1 & 0 & 0 & 0 \\ 0 & \cos (A) & -\sin (A) & 0 \\ 0 & \sin (A) & \cos (A) & 0 \\ 0 & 0 & 0 & 1\end{array}\right]\left[\begin{array}{c}0 \\ 0 \\ -L \\ 1\end{array}\right]=\left[\begin{array}{c}-L \sin (A) \sin (C) \\ L \sin (A) \cos (C) \\ -L \cos (A) \\ 1\end{array}\right]$
$T_{n}=\left[\begin{array}{cccc}\cos (C) & -\sin (C) & 0 & 0 \\ \sin (C) & \cos (C) & 0 & 0 \\ 0 & 0 & 1 & 0 \\ 0 & 0 & 0 & 1\end{array}\right]\left[\begin{array}{cccc}1 & 0 & 0 & 0 \\ 0 & \cos (A) & -\sin (A) & 0 \\ 0 & \sin (A) & \cos (A) & 0 \\ 0 & 0 & 0 & 1\end{array}\right]\left[\begin{array}{l}0 \\ 0 \\ 1 \\ 1\end{array}\right]=\left[\begin{array}{c}\sin (A) \sin (C) \\ -\sin (A) \cos (C) \\ \cos (A) \\ 0\end{array}\right]$

In the case of kinematic structure, when the workpiece done translational movements in the $X Y Z$ axes, and the tool can only rotate about the axis $\mathrm{A}$ and $\mathrm{C}$ the final point $\mathrm{On}$ of the tool orientation vector $\mathrm{Tn}$ is:

$$
\begin{gathered}
0_{n}=0_{r}=\left[\begin{array}{c}
-L \sin (A) \sin (C) \\
L \sin (A) \cos (C) \\
-L \cos (A) \\
1
\end{array}\right] \\
T_{n}=T_{r}=\left[\begin{array}{c}
\sin (A) \sin (C) \\
-\sin (A) \cos (C) \\
\cos (A) \\
0
\end{array}\right]
\end{gathered}
$$

In the case of kinematic structure, when the workpiece performs translational motion along the axis $Y$, and $Z$ tool will perform translational motion along the $Y$ axis, rotation about axes $A$ and $C$ can be $O_{n}$ and $T_{n}$ defined as:

$$
0_{r}=0_{n}+P_{r}=\left[\begin{array}{c}
X-L \sin (A) \sin (C) \\
L \sin (A) \cos (C) \\
-L \cos (A) \\
2
\end{array}\right]
$$

Where:

$$
P_{r}=\left[\begin{array}{llll}
1 & 0 & 0 & X \\
0 & 1 & 0 & 0 \\
0 & 0 & 1 & 0 \\
0 & 0 & 0 & 1
\end{array}\right]\left[\begin{array}{l}
0 \\
0 \\
0 \\
1
\end{array}\right]=\left[\begin{array}{l}
X \\
0 \\
0 \\
1
\end{array}\right]
$$

Vector $T_{r}$ depends only on the rotation of the tool, so its value will be for all machines RR (Rotation Rotation) constants:

$$
T_{n}=T_{r}=\left[\begin{array}{c}
\sin (A) \sin (C) \\
-\sin (A) \cos (C) \\
\cos (A) \\
0
\end{array}\right]
$$

In RRTTT' structure, ie. when the tool will perform translational motion in only one of the axes, for example. $X$ axis point 0 be expressed as:

$$
T_{n}=T_{r}=\left[\begin{array}{c}
\sin (A) \sin (C) \\
-\sin (A) \cos (C) \\
\cos (A) \\
0
\end{array}\right]
$$

And the description of the vector $\mathrm{P}_{\mathrm{r}}$ :

$$
0_{r}=0_{n}+P_{r}=\left[\begin{array}{c}
-L \sin (A) \sin (C) \\
Y+L \sin (A) \cos (C) \\
Z-L \cos (A) \\
2
\end{array}\right]
$$

If machined component will not make any moves and the tool will move in all five axes $(X, Y, Z, A, C) 0_{r}$ point is expressed as:

$$
P_{r}=\left[\begin{array}{llll}
1 & 0 & 0 & 0 \\
0 & 1 & 0 & Y \\
0 & 0 & 1 & 0 \\
0 & 0 & 0 & 1
\end{array}\right]\left[\begin{array}{llll}
1 & 0 & 0 & 0 \\
0 & 1 & 0 & 0 \\
0 & 0 & 1 & Z \\
0 & 0 & 0 & 1
\end{array}\right]\left[\begin{array}{l}
0 \\
0 \\
0 \\
1
\end{array}\right]=\left[\begin{array}{l}
0 \\
Y \\
Z \\
1
\end{array}\right]
$$

And the description of the vector $\mathrm{P}_{\mathrm{r}}$ :

$$
0_{r}=0_{n}+P_{r}=\left[\begin{array}{c}
X-L \sin (A) \sin (C) \\
Y+L \sin (A) \cos (C) \\
Z-L \cos (A) \\
2
\end{array}\right]
$$

\section{Conclusions}

The paper presents a methodology for features extracting from CAD data and description of the kinematic structure of CNC machine tools. As mentioned above, the goal is to get a group of data which will serve as input to the classification system. This classification system will have the task associate a specific components to specific 
CNC machine. To be able to classify the component from the view its production on $\mathrm{CNC}$ machines is necessary to know the kinematic structure of the CNC machines and appropriate way to describe these structures. But itself description of kinematic structures is not sufficient and will serve as input for the inverse kinematics theory. The role of the inverse kinematics theory will determine the number of joints and angles of rotation. This data could be used for software designed in [Michalik 2012].

A similar description of the of kinematic structure was introduced in [XIE, 2009] where the author described the kinematic structure of the type "universal head".

\section{References}

[Bohez 2004] Bohez, E. L. J. Five-axis milling machine tool kinematic chain design and analysis. In: International Journal of Machine Tools \& Manufacture, vol. 42, 2002, pp. 505-520, 2004

[Chang 1991] Chang, T. C., Wysk, R. A. Computer-Aided Manufacturing, Prentice-Hall, Englewood Cliffs, NJ, 1991

[Cunningham 1988] Cunningham, J. J., Dixon, J. R., Designing with features: the origin of features., In: Computers in Engineering Conf., CIE, San Francisco CA, 1988

[Fabian 2010] Fabian, M., Spisak, E., Seminsky, J., Senderska, K., Mares, A., Izol, P. CAM parameters settings and NC milled surface quality. In: Annals of DAAAM for 2010 \& Proceedings of the $27^{\text {th }}$ international DAAAM symposium. Vienna: DAAAM International, pp. 391-392. ISBN 978-3-901509-73-5 ISSN 1726-9679, 2010

[Michalik 2012] Michalik, P., Zajac, J., Duplak, J., Pivovarnik, A. CAM Software Products for Creation of Programs for CNC
Machining. In: Lecture Notes in Electrical Engineering. ISSN 1876-1100. - Vol. 141, 2012

[Salomons 1988] Salomons, O. W., Kals, H.J.J. Review of research in feature-based design, In: J. Manuf. Syst. vol. 12, pp. 113-132, 1993 [Shu-chu 1995] Shu-Chu, L., Gonzales, M. Development of an automatic part feature extraction and classification system taking $C A D$ data as input. In: Computers in Industry, vol. 29, pp. 137-150, 1995

[Xie 2009] Xie, C., Zhang, W. M., He, X. Y. Kinematic analysis and post-processing algorithm research for 5 -axis CNC machine tools with a universal head. In Industrial Engineering and Engineering Management, pp. 2309-2313, 2009

\section{Contacts}

assoc. prof. Ing. Peter Pokorny, PhD.

Slovak University of Technology

Faculty of Materials Science and Technology in Trnava

Institute of Production Technologies

Department of Machining and Assembly

J. Bottu 25, 91724 Trnava, Slovak Republic

tel.: +421905906 371, e-mail: peter_pokorny@stuba.sk

Vladimir Simna, MSc.

Slovak University of Technology

Faculty of Materials Science and Technology in Trnava

Institute of Production Technologies

Department of Machining and Assembly

J. Bottu 25, 91724 Trnava, Slovak Republic

tel.: +421 902734 179, e-mail: vladimir.simna@stuba.sk 